\title{
GAIA Level 1 Neonatal Invasive Bloodstream Infection
}

National Cancer Institute

\section{Source}

National Cancer Institute. GAIA Level 1 Neonatal Invasive Bloodstream Infection. NCI

Thesaurus. Code C127984.

GAIA Level 1 Neonatal Invasive Bloodstream Infection is defined by two criteria: first, identification of a recognized pathog en from a normally sterile site using a validated method; second, if a normally non-pathogenic organism is isolated from blood cultures the following two requirements must be met: a) The cultures must be obtained from two different sites OR taken at two different times; b) One or more of the level 2 criteria must be present. 